\title{
Lessons learned from Radial Artery Database International ALliance (RADIAL)
}

\author{
Mario F. L. Gaudino ${ }^{1}$, Jeremy R. Leonard ${ }^{1}$, David P. Taggart ${ }^{2}$ \\ ${ }^{1}$ Department of Cardiothoracic Surgery, Weill Cornell Medicine, New York, NY, USA; ${ }^{2}$ Department of Cardiovascular Surgery, University of \\ Oxford, Oxford, UK \\ Correspondence to: Mario F. L. Gaudino, MD, FEBCTS. Department of Cardiothoracic Surgery, Weill Cornell Medicine, 525 East $68^{\text {th }}$ Street, New \\ York, NY 10065, USA. Email: mfg9004@med.cornell.edu.
}

\begin{abstract}
Coronary artery bypass graft (CABG) surgery was first performed in the 1960s. As the surgery has evolved, there has been a growing interest in the use of multiple arterial grafts in CABG. Since the re-introduction of the radial artery (RA) to clinical use as a bypass conduit in the 1990s, there have been several randomized controlled trials (RCTs) which have compared saphenous vein graft (SVG) conduits to RA use in CABG. While most trials have shown improved patency of the RA, none of them have been able to demonstrate a survival benefit using the arterial conduit. In this review, we examine the existing RCTs on the subject. We then look at our solution to the decades-old inquiry regarding the RA compared with the SVG. The Radial Artery Database International ALliance (RADIAL) project is an individual patient-level meta-analysis developed to adequately power a study to assess if the RA has superior clinical outcomes compared with the SVG. We describe the process by which this investigation was conducted and the collaboration necessary to achieve success.
\end{abstract}

Keywords: Radial artery (RA); coronary artery bypass graft surgery (CABG surgery); multiple arterial grafts; patient-level meta-analysis

Submitted Feb 01, 2018. Accepted for publication Mar 15, 2018.

doi: $10.21037 /$ acs.2018.03.15

View this article at: http://dx.doi.org/10.21037/acs.2018.03.15

\section{Introduction}

Coronary artery bypass graft (CABG) surgery was first successfully performed in humans in the 1960s (1). Since then, there has been much debate over the appropriate choice and use of bypass conduits. Sabiston used autologous vein grafts beginning in 1962 and the first sutured internal mammary graft was reportedly completed by Kolessov in 1964 (2). In 1986, Loop and colleagues showed a significant improvement in long-term survival following CABG when an internal mammary artery (IMA), rather than a saphenous vein graft (SVG), was used to graft the left anterior descending artery (LAD) (3).

Following the re-introduction of the radial artery (RA) in the 1990s as an additional arterial conduit in CABG, a large volume of supporting evidence has been collected (4). To date, several randomized controlled trials (RCTs) have compared SVG to RA use in CABG. While most trials have shown improved patency of the RA, none of them have been able to demonstrate a survival benefit using the arterial conduit. However, none of the existing RCTs were appropriately powered to detect a difference in clinical outcome.

With this framework in place, the beginnings of the Radial Artery Database International ALliance (RADIAL) project were formed.

\section{Pre-existing individual RCTs}

\section{Radial Artery Versus Saphenous Vein Patency (RSVP) study}

The RSVP trial 5-year angiographic follow-up study was published in Circulation in 2008 (5). The study investigators enrolled 142 patients at the Royal Brompton Hospital in the UK. The patients were randomized to receive either the RA 
or a SVG as the conduit to bypass the left circumflex artery. At 5-year follow-up, 134/142 (94.4\%) patients were alive and eligible for repeat angiography. However, angiography was only performed in 103/134 (76.9\%) of them. The late follow-up results showed $98.3 \%$ of RA grafts remained patent compared with $86.4 \%$ of SVGs $(\mathrm{P}=0.04)$.

While the results allow for the conclusion that the RA offers better patency at 5-year follow-up, nothing definitive can be said about clinical outcomes based on this study. The authors acknowledge, "the only robust clinical outcome assessed was mortality". The study was powered at $80 \%$ to detect a $15 \%$ absolute difference in angiography patency at 5 years and was not designed to assess clinical outcomes.

The 30-day mortality in the study was comprised of a single patient in the SVG subgroup. Furthermore, there were no differences in in-hospital morbidity except for delayed wound healing in the SVG group (16\% vs. $5 \%, \mathrm{P}=0.03)$. The study also reports no adverse forearm complications following RA harvest in the patient sample. At the follow-up point of 5 years, there was no statistically significant difference in survival between the two groups.

From this RCT, we derived several salient points. First (and most importantly), the study does not have enough subjects and was not powered to detect significant difference in clinical end-points. Second, the one significant clinical outcome from the study, delayed wound healing in the SVG harvest group, is in line with previous investigation on the subject (6). Finally, given the high overall survival rate, it seems likely that a longer follow-up period would have been necessary to determine if there was a difference in survival benefit between the two groups.

\section{Stand-in-Y Mammary Study}

In 2009, Nasso and colleagues published their results comparing RA and SVG conduits as a subset of the Stand-in-Y Mammary Study conducted in Italy (7). They sought to determine if multiple arterial grafts offered a benefit over a single arterial conduit and to investigate if a certain configuration of arterial conduits offered a survival advantage. A total of 803 patients were enrolled in this prospective, randomized trial. The subset which compared RA to SVG results consisted of 409 patients. The protocol necessitated that the RA be harvested from the patient's non-dominant arm and was always used as the second-choice conduit to revascularize the secondary coronary target [after the left IMA (LIMA) to the LAD]. Furthermore, the study specified that the RA could only be anastomosed to a target with greater than 70 percent stenosis.

At a mean follow-up time of 2 years, the group which received arterial grafts (including RA grafts either as free aortocoronary grafts or $\mathrm{Y}$ grafts) had all-cause mortality of $3.2 \%$, while the group which only received SVG secondary bypasses had all-cause mortality of $4.9 \%(\mathrm{P}=0.33)$. On Kaplan-Meier analysis, there was no statistically significant overall survival advantage in any of the study groups.

In contrast, the arterial revascularization groups had a significantly higher rate of freedom from adverse cardiac events when compared with the SVG group $(\mathrm{P}<0.001)$. Furthermore, the graft occlusion rate and need for percutaneous angioplasty were significantly higher in the SVG group $(\mathrm{P}<0.001$ and $\mathrm{P}=0.048$, respectively). Finally, the rate of recurrent angina was higher amongst patients in the SVG group compared with the arterial revascularization groups $(\mathrm{P}<0.001)$.

The investigators acknowledge that the study cannot answer some of the fundamental questions we are asking, due to an insufficient sample size. While they postulate that the benefits of two arterial conduits might be relevant not only in mid-term follow-up but also in the immediate postoperative setting with regards to incidence of stroke, this difference was non-significant with the Stand-in-Y sample size ( $3 \%$ vs. $4.9 \%, \mathrm{P}=0.065)$.

In this RCT we again see the limitations of sample size and a study with insufficient power to detect potentially important differences in clinical outcomes. There were also slightly different study criteria involved in this trial (when compared with the RSVP investigation). Notably in this study, the second-choice conduit did not have to be to the left circumflex territory (although it was in the majority of cases), whereas this was mandatory in RSVP. Also, this protocol specified that the RA could only be harvested from the non-dominant arm, potentially limiting the number of people eligible for the randomization. Finally, with a followup of only 2 years, we are again limited from drawing any long-term conclusions regarding survival advantage.

\section{Radial Artery Patency and Clinical Outcomes (RAPCO)}

The RAPCO trial, undertaken in Australia, was a randomized controlled trial designed to assess the longterm patency and clinical outcomes of the RA, the right internal thoracic artery (RITA) and the saphenous vein when grafted to the second-choice target vessel $(8,9)$. In this two-arm trial, one of the arms offered a direct comparison of the RA and SVG in CABG. A total of 619 patients 
participated in the study. Of these, 225 patients older than 70 years of age were randomized to receive the RA or the SVG as the second bypass conduit. At mean follow-up of 6 years, the patency rates of the RA and SVG were similar $(\mathrm{P}=0.54)$. There was no difference in actuarial survival between the two groups and there was no difference in event-free survival. The SVG group did have higher rates of target vessel revascularization (3.6\% vs. $0.9 \%)$ but again (probably due the small sample size), this difference did not reach statistical significance.

While the mid-term results of the RAPCO trial were neutral, the 10-year still unpublished results, presented at the 2016 annual meeting of the American Association for Thoracic Surgery (AATS), showed better RA patency when compared to the SVG $(\mathrm{P}=0.03)$.

In this trial, the primary endpoint was, once again, an angiographic measure. Thus, even in the final 10-year analysis it is unlikely that the investigators will be able to comment on any survival benefit.

\section{Veteran Affairs (VA) Study}

A study conducted at $11 \mathrm{VA}$ medical centers and published in 2011 aimed to compare the 1-year angiographic patency of RA grafts to SVGs in patients undergoing elective CABG (10). While the trial did randomize 757 patients over 6 years, the primary endpoint occurred after only 1 year, not permitting an analysis of long-term outcomes. In the investigation, all patients received the standard LIMA to LAD anastomosis followed by the randomized study conduit.

The study was designed to be powered at $90 \%$ to detect a difference in patency at 1-year. Angiographic follow-up was completed by $73 \%$ of patients. In the study, approximately $88 \%$ of cases were performed on pump, with no difference between the two groups. Of note, there was no difference in operative mortality between the SVG group and the RA group $(0.5 \%$ vs. $0.8 \%, \mathrm{P}=0.69)$. The only statistically significant post-operative complication was an increased risk of bleeding in the SVG group ( $3.3 \%$ vs. $0.8 \%, \mathrm{P}=0.03$ ). At 1-year follow-up, the graft patency was $89 \%$ in both groups $(\mathrm{P}=0.82)$. Furthermore, there was no difference in complications between the two groups at 1 year.

While these results do not demonstrate that the RA is superior to the SVG, limited follow-up certainly can account for the lack of difference. This study only reported a follow-up time of 1 year. Previously, it has been demonstrated that results between arterial and vein conduits diverge beginning at 4 years post-operatively (11).

\section{Radial Artery Patency Study (RAPS)}

The RAPS involved 510 patients from nine centers across Canada. In 2012, the investigators published their results with a mean follow-up of 7.7 years $(12,13)$. At this time, they found a significant difference in patency between the RA and the SVG $(88.0 \%$ vs. $80.3 \%, \mathrm{P}=0.03)$. This study, unlike the other RCTs, used within-patient randomization. Patients received either the RA to the right coronary artery and the SVG to the left circumflex territory or the inverse (14). Overall mortality at late follow-up was $11.5 \%$ with $20.2 \%$ of patients having experienced a major adverse cerebral and cardiac event (MACCE).

This study, although of interest due to the within-patient randomization, does not aid a conclusion about clinical outcomes. The RAPS investigators directly acknowledge this in the publication of their mid-term results stating, "although the design of this trial is powerful for assessing angiographic outcomes, because all patients receive both study grafts, it is weak for the evaluation of clinical outcomes" (12). However, based on the analysis, the study does conclude that "the benefit of the RA compared with a vein graft persists over 7.5 years".

\section{Korean off-pump CABG (OPCABG)}

A group led by Dr. Yoo at the Severance Hospital in Seoul, South Korea published a study in 2012 which aimed to evaluate RA and SVG conduits and their use in OPCABG (15). This was a relatively small trial which randomized patients to receive either a RA conduit or a SVG as the second-choice graft. Further restricting the study, all patients enrolled had to be over the age of 70 and had to undergo only OPCABG.

Once again, the primary endpoint was angiographic at 1 -year following surgery. Overall patency of the 60 patients achieved a rate of $96.3 \%$, with the RA group at $97.4 \%$ and the SVG group at $94.7 \%(\mathrm{P}=0.44)$. Unsurprisingly, there was no difference in overall survival between the two study arms.

Importantly, this study had a specifically defined operative strategy when compared with the other RCTs. All RA or SVG conduits were carried off from the insitu LIMA. None of the bypass vessels had a proximal anastomosis on the aorta.

\section{Serbian randomized trial}

In 2015, the group from the Dedinje Cardiovascular 
Institute in Belgrade, Serbia published a series with the longest follow-up to date (16). They found that in a group of 200 patients randomized to either the RA or SVG as the secondary bypass conduit, there was no significant difference in absolute survival at 8 -year follow-up. While RA graft patency was $92 \%$ compared with $86 \%$ for the SVG group, these findings were not statistically significant $(\mathrm{P}=0.67)$. Furthermore, they found no difference in clinical outcomes between the two groups. Both arms had comparable occurrence of cerebrovascular events $(\mathrm{P}=0.65)$, perioperative myocardial infarction $(\mathrm{P}=0.70)$, atrial fibrillation $(\mathrm{P}=0.75)$, pleural effusion $(\mathrm{P}=0.77)$ and sternal dehiscence $(\mathrm{P}=0.56)$.

In this study, it is important to note that RA grafts were only used to the right coronary artery $17 \%$ of the time, with the majority of RA bypasses going to the left circumflex territory. As a further restriction, the authors note that, "[the] $R A$ graft was never placed to the right coronary artery or diagonal branch if they were previously occluded".

The reason for the lack of clinical and angiographic difference in this study is likely the small sample size. Despite the long follow-up, with only 200 patients the study was not powered to detect even large differences in clinical outcomes.

In the discussion, the authors note the difficulty apparent when deciding which bypass conduit to utilize. They state, "Since it appears that [the] $R A$ is not superior in terms of clinical outcome to the vein grafts for revascularization of the right coronary artery, we usually use $R A$ for revascularization of the left side system...the main target for [the] $R A$ graft is [the] obtuse marginal artery with at least $80 \%$ stenosis". Thus, they conclude that the RA, rather than SVG, should be used as the secondary conduit to the left side of the heart but are inconclusive about the benefits to the right coronary system.

\section{The RADIAL project}

In 2015, the principal investigators (PIs) of the RADIAL project (MF Gaudino, DP Taggart) sought to provide evidence-based outcomes on the clinical results of the use of the RA compared to the SVG in CABG. In reviewing the prior RCTs which had been completed, it was clear that all the trials had primary angiographic endpoints and did not have sufficient power to detect even moderate differences in clinical outcome. While some of the RCTs showed trends toward clinical superiority of the RA, none reach statistical significance.

Thus, the problem became apparent. Either we needed a new randomized controlled trial comparing the RA and the SVG which would be sufficiently powered to detect a clinical difference (major cardiac event and possibly mortality), or we needed some other way to have sufficient statistical power. Prior to tackling this problem, an appropriate sample size calculation was needed.

The starting point for this calculation was the Synergy between PCI with Taxus and Cardiac Surgery (SYNTAX) trial, which published 5-year results in 2009 (17). Given that the primary outcomes of SYNTAX were clinical, it seemed an obvious choice for comparison. In patients with triple-vessel disease who underwent CABG, incidence of death at 5 -years was approximately $12 \%$ and the incidence of MACCE was approximately $27 \%$. In order to detect a $25-30 \%$ reduction in mortality at a confidence level of $90 \%$, somewhere between 3,000 and 4,000 patients would be needed. In looking at MACCE, to detect the same difference, between 1,200-1,700 patients would be needed. Unfortunately, the largest published RCT on the RA involved only 757 patients.

With the necessary sample size calculated, the RADIAL investigators devised a plan to achieve an appropriately powered study. The idea was to combine the data from all the pre-existing RCTs in order to determine if the use of the RA was associated with a significant reduction in major cardiac events. A traditional systematic review would not have accomplished this due to the composite nature of the outcome. Thus, the way to garner the most relevant and necessary data was to examine the data at the level of the individual patients. By combining all of the RCTs, the total subject number would have been sufficient to detect a difference in cardiac events.

\section{Patient level meta-analysis}

In April 2016, at the annual meeting of the AATS in Baltimore, the primary investigators of the individual RCTs agreed to participate in the patient-level meta-analysis. It was at this point that project took on the name RADIAL (Figure 1). Appropriate permission and ethics review had to be obtained to share individual patient-level data from each trial. All data had to be de-identified prior to input into the combined database. Updated follow-up information compared to the original publication were requested for all trials.

The primary objective of RADIAL was to evaluate the clinical effects of using the RA instead of the SVG as the second graft in an individual participant data meta-analysis 


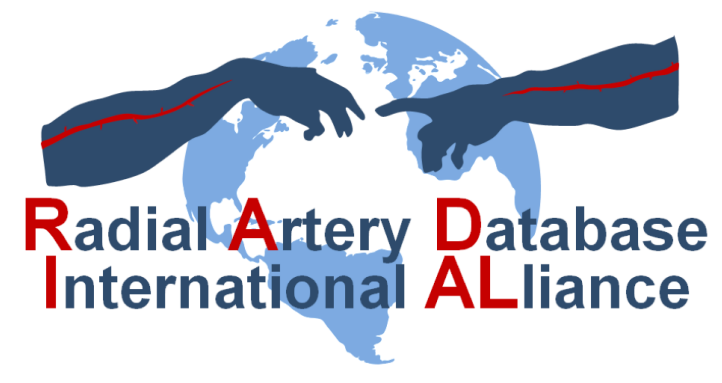

Figure 1 The RADIAL logo.

of all RCTs on the topic. Additionally, we stipulated predefined sub-analysis in specific subsets including: female patients, diabetic patients, obese patients and patients with non-dialysis dependent renal failure. The secondary objective of RADIAL was to evaluate the clinical effects of using the RA instead of the SVG as the second graft in a large propensity-matched database and to then compare the results of individual RCTs to those propensity-matched studies.

Participation in the RADIAL project had to be approved by the lead study centers and patient data then had to be further de-identified to ensure the confidentiality of all study participants. After access to the individual patient data from all the RCTs and collaboration with all primary investigators of the trials, the raw data collected was combined into a single, large database under the direction and execution of the RADIAL investigators.

Definitions used in the single trials were evaluated for homogeneity. In general, definitions from the RAPCO trial were used as the references. In case of major discordance between definitions, a further request of hard data was performed in order to re-categorize patients using the RAPCO definitions. Homogenous data was then joined in a common database by a team of clinical information analysts; data collection was validated by means of external and internal control. Data collection and editing was based at Weill Cornell Medicine in New York City.

When available, preoperative and follow-up angiographies were collected and subjected to blind reevaluation in an independent core lab.

Statistical analysis was carried out by an independent group of statisticians with access to the final version of the joined database. Statistical analysis was mainly performed at the Bristol Heart Institute.

Finally, in addition to the primary investigators of the individual RCTs, the RADIAL project has recruited experts

\begin{tabular}{|c|c|}
\hline Name & Affiliation \\
\hline Mario Gaudino & Weill Cornell Medicine, New York, US (PI) \\
\hline David P. Taggart & University of Oxford, United Kingdom (PI) \\
\hline Umberto Benedetto & Bristol Heart Institute, Bristol, UK \\
\hline Brian Buxton & University of Melbourne, Australia \\
\hline Stephen Fremes & University of Toronto, Toronto, Canada \\
\hline Leonard N. Girardi & Weill Cornell Medicine, New York, NY, USA \\
\hline Steven Goldman & University of Arizona, Tucson, USA \\
\hline Robert Habib & $\begin{array}{l}\text { The Society of Thoracic Surgeons Research } \\
\text { Center, Chicago, USA }\end{array}$ \\
\hline David L. Hare & $\begin{array}{l}\text { Department of Medicine, University of } \\
\text { Melbourne, Australia }\end{array}$ \\
\hline Philip Hayward & $\begin{array}{l}\text { Victorian Heart Centre, Richmond Vic, } \\
\text { Australia }\end{array}$ \\
\hline William L. Holman & $\begin{array}{l}\text { Alabama VA Medical Center, Birmingham, } \\
\text { USA }\end{array}$ \\
\hline Neil Moat & $\begin{array}{l}\text { Royal Brompton \& Harefield Trust, London, } \\
\text { UK }\end{array}$ \\
\hline Claudio Muneretto & $\begin{array}{l}\text { University of Brescia Medical School, } \\
\text { Brescia, Italy }\end{array}$ \\
\hline Giuseppe Nasso & Anthea Hospital, Bari, Italy \\
\hline Miodrag Peric & $\begin{array}{l}\text { Dedinje Cardiovascular Institute and Belgrade } \\
\text { University School of Medicine, Belgrade, } \\
\text { Serbia }\end{array}$ \\
\hline Ivana Petrovic & $\begin{array}{l}\text { Dedinje Cardiovascular Institute and Belgrade } \\
\text { University School of Medicine, Belgrade, } \\
\text { Serbia }\end{array}$ \\
\hline John D. Puskas & $\begin{array}{l}\text { Icahn School of Medicine at Mount Sinai, } \\
\text { New York, NY, USA }\end{array}$ \\
\hline $\begin{array}{l}\text { Elfriede } \\
\text { Ruttmann-Ulmer }\end{array}$ & University Hospital Innsbruck, Austria \\
\hline Thomas A. Schwann & University of Toledo, Toledo, OH, USA \\
\hline James Tatoulis & $\begin{array}{l}\text { Royal Melbourne Hospital, Melbourne, } \\
\text { Australia }\end{array}$ \\
\hline Robert Tranbaugh & Weill Cornell Medicine, New York, NY, USA \\
\hline Kyung Jong Yoo & $\begin{array}{l}\text { Yonsei University College of Medicine, Seoul, } \\
\text { South Korea }\end{array}$ \\
\hline
\end{tabular}

at the forefront of the field to contribute to the writing group and for data analysis (Table 1).

\section{Conclusions}

In order to achieve results that change the standard of 
care, extensive and arduous work must be carried out. The vision of RADIAL is to overcome the limitations of sample size and statistical power to answer a decades-old query. By combining all the RCTs which compare RA and SVG use during CABG, we have aimed to determine if the RA offers an advantage in clinical outcomes when compared with the SVG. Using individual patient-level data, we will have sufficient sample size to detect significant differences, should they exist. Throughout the RADIAL project, surgeons from across the world have collaborated in hopes of benefiting our future patients with the best possible surgical options. We have shown with this undertaking that patient-level meta-analysis is not only possible, but is vital to utilize when we reach an impasse in inquiry. When the final results are published, the RADIAL project will be a landmark study in the evolving universe of arterial revascularization in CABG.

\section{Acknowledgements}

None.

\section{Footnote}

Conflicts of Interest: The authors have no conflicts of interest to declare.

\section{References}

1. Buxton BF, Galvin SD. The history of arterial revascularization: from Kolesov to Tector and beyond. Ann Cardiothorac Surg 2013;2:419-26.

2. Kolessov VI. Mammary artery-coronary artery anastomosis as method of treatment for angina pectoris. J Thorac Cardiovasc Surg 1967;54:535-44.

3. Loop FD, Lytle BW, Cosgrove DM, et al. Influence of the internal-mammary-artery graft on 10-year survival and other cardiac events. N Engl J Med 1986;314:1-6.

4. Acar C, Jebara VA, Portoghese M, et al. Revival of the radial artery for coronary artery bypass grafting. Ann Thorac Surg 1992;54:652-9; discussion 659-60.

5. Collins P, Webb CM, Chong CF, et al. Radial Artery Versus Saphenous Vein Patency Randomized Trial: Five-Year Angiographic Follow-Up. Circulation 2008;117:2859-64.

6. Hata M, Raman, Matalanis G, et al. Post harvest wound infection and patient's perception: comparative study between radial artery and saphenous vein harvest sites.
Ann Thorac Cardiovasc Surg 2002;8:97-101.

7. Nasso G, Coppola R, Bonifazi R, et al. Arterial revascularization in primary coronary artery bypass grafting: Direct comparison of 4 strategies-Results of the Stand-in-Y Mammary Study. J Thorac Cardiovasc Surg 2009;137:1093-100.

8. Hayward PA, Buxton BF. The Radial Artery Patency and Clinical Outcomes trial: design, intermediate term results and future direction. Heart Lung Circ 2011;20:187-92.

9. Hayward PA, Buxton BF. Mid-term results of the Radial Artery Patency and Clinical Outcomes randomized trial. Ann Cardiothorac Surg 2013;2:458-66.

10. Goldman S, Sethi GK, Holman W, et al. Radial Artery Grafts vs Saphenous Vein Grafts in Coronary Artery Bypass Surgery: A Randomized Trial. JAMA 2011;305:167-74.

11. Gaudino M, Crea F, Cammertoni F, et al. The Radial Artery: A Forgotten Conduit. Ann Thorac Surg 2015;99:1479-85.

12. Deb S, Cohen EA, Singh SK, et al. Radial Artery and Saphenous Vein Patency More Than 5 Years After Coronary Artery Bypass Surgery: Results From RAPS (Radial Artery Patency Study). J Am Coll Cardiol 2012;60:28-35.

13. Desai ND, Cohen EA, Naylor CD, et al. A Randomized Comparison of Radial-Artery and Saphenous-Vein Coronary Bypass Grafts. N Engl J Med 2004;351:2302-9.

14. Fremes SE. Multicenter radial artery patency study (RAPS). Study design. Control Clin Trials. 2000;21:397-413.

15. Song SW, Sul SY, Lee HJ, et al. Comparison of the radial artery and saphenous vein as composite grafts in off-pump coronary artery bypass grafting in elderly patients: a randomized controlled trial. Korean Circ J 2012;42:107-12.

16. Petrovic I, Nezic D, Peric M, et al. Radial artery vs saphenous vein graft used as the second conduit for surgical myocardial revascularization: long-term clinical follow-up. J Cardiothorac Surg 2015;10:127.

17. Serruys PW, Morice MC, Kappetein AP, et al. Percutaneous coronary intervention versus coronary-artery bypass grafting for severe coronary artery disease. N Engl J Med 2009;360:961-72.

Cite this article as: Gaudino MF, Leonard JR, Taggart DP. Lessons learned from Radial Artery Database International ALliance (RADIAL). Ann Cardiothorac Surg 2018;7(5):598603. doi: $10.21037 /$ acs.2018.03.15 